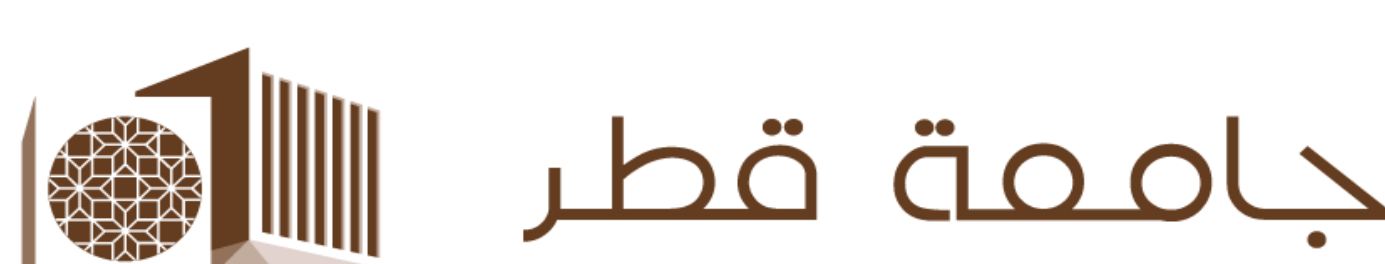
QATAR UNIVERSITY

Graduate Students, Energy and Environment
TOWARDS DEVELOPING A HYBRID NONLINEAR VIBRATION ENERGY

HARVESTER FOR REMOTE SENSING APPLICATIONS:

A DESIGN AND OPTIMIZATION STUDY

Muhammad Hafizh1 ${ }^{1}$ Asan G. A. Muthalif2*

Department of Mechanical and Industrial Engineering, College of Engineering, Qatar University, Doha, Qatar

drasan@qu.edu.qa

\section{INTRODUCTION}

Qatar has pipeline networks spanning thousands of kilometers that requires strict monitoring and inspections to prevent leakage or catastrophic failures.

- Real - time monitoring of information requires an integrated system of sensors wireless communication routers and power supply to relay information.

Lifespan degradation and periodic replacement would make the use of batteries non-sustainable and unfeasible for condition-monitoring and leakage detection. Vibration-based energy (VEH) harvesting is an emerging topic of research that can utilize ambient surroundings in powering MEMS devices and is selfsustaining

Piezoelectric and electromagnetic energy harvesting are promising as they are very cost-effective, versatile in application and has a high-power density

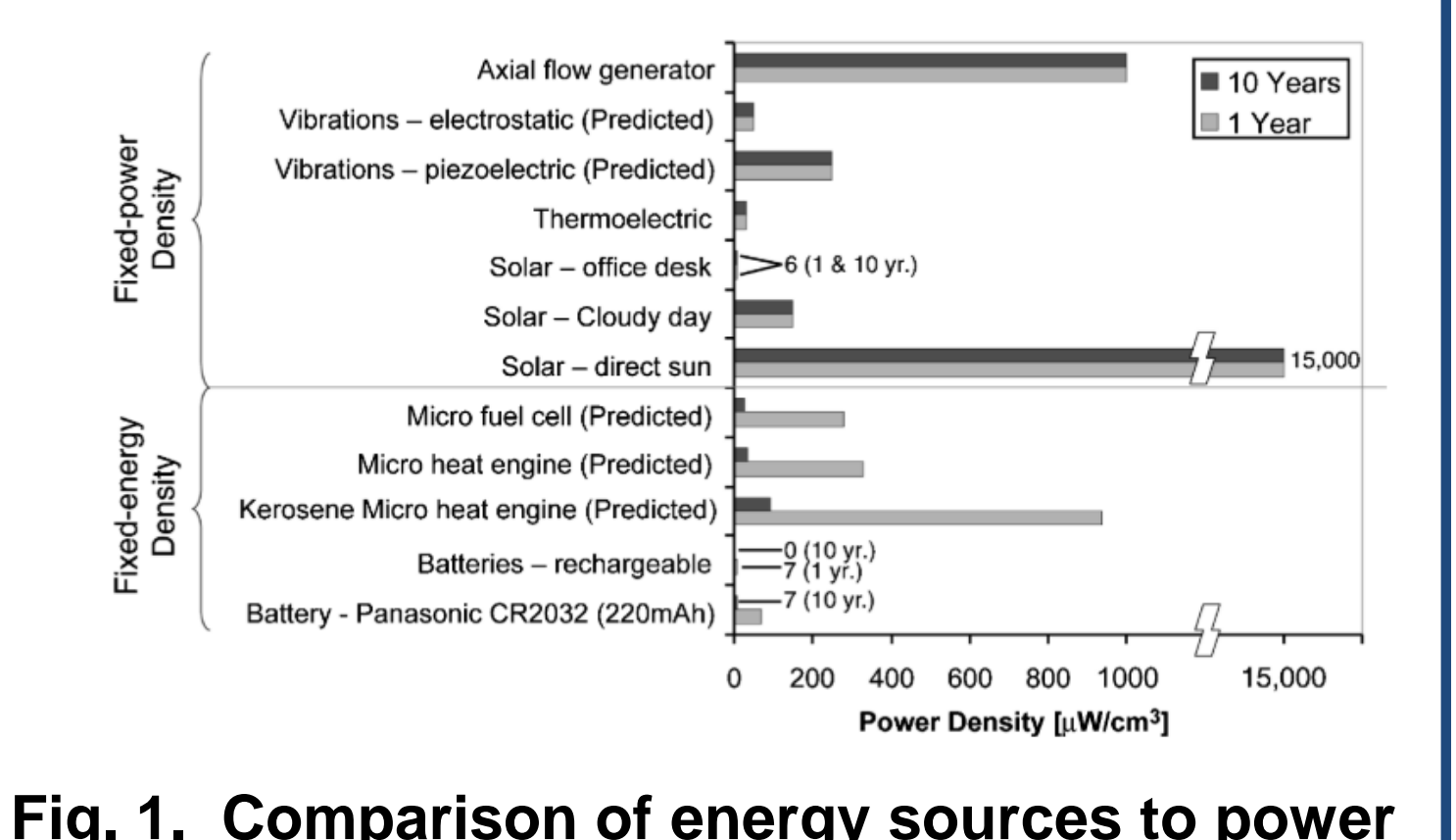
Fig. 1. Comparison of energy sources to power
integrated systems and MEMS devices [1] Vortex-induced vibration (VIV) is a widely adopted method of VEH that takes advantage of periodic oscillations in vortices and synchronization of flow [2].

A compact hybrid piezoelectric-electromagnetic vibration-based energy harvester that is excited by fluid flow is proposed.

Significance of current work:

- The outcome of this work can be extended to harvest energy from various vibration sources like machinery, roads, sea waves and railway tracks.

- Nonlinear energy scavengers can introduce a more reliable and sustainable pipeline inspection system with improved characteristics and performance.

- Bandwidth enhancement of hybrid energy harvesters can provide a more robust solution to variable input vibration sources in active pipelines.

- Work in pipeline can be extended to wind-flow in windy environments.

\section{METHODOLOGY}

1. Study the architecture of energy harvesting mechanisms in literature and analyze vibration and structural dynamics of multi-dynamic oscillations by considering structural and geometrical nonlinearities under resonance

2. Develop the fluid-structure interaction (FSI) that can be developed using vortex-induced vibration (VIV) and to develop a generalized Multiphysics model

3. Solve the FSI nonlinear structural mechanics model by modelling under resonance for nonlinear excitation of VIV using numerical methods

4. Design optimizations of energy harvester investigated using finite element modelling (FEM): (a) broadband harvester; (b) hybrid harvester

5. Perform experimental tests in a controlled and real-time environment

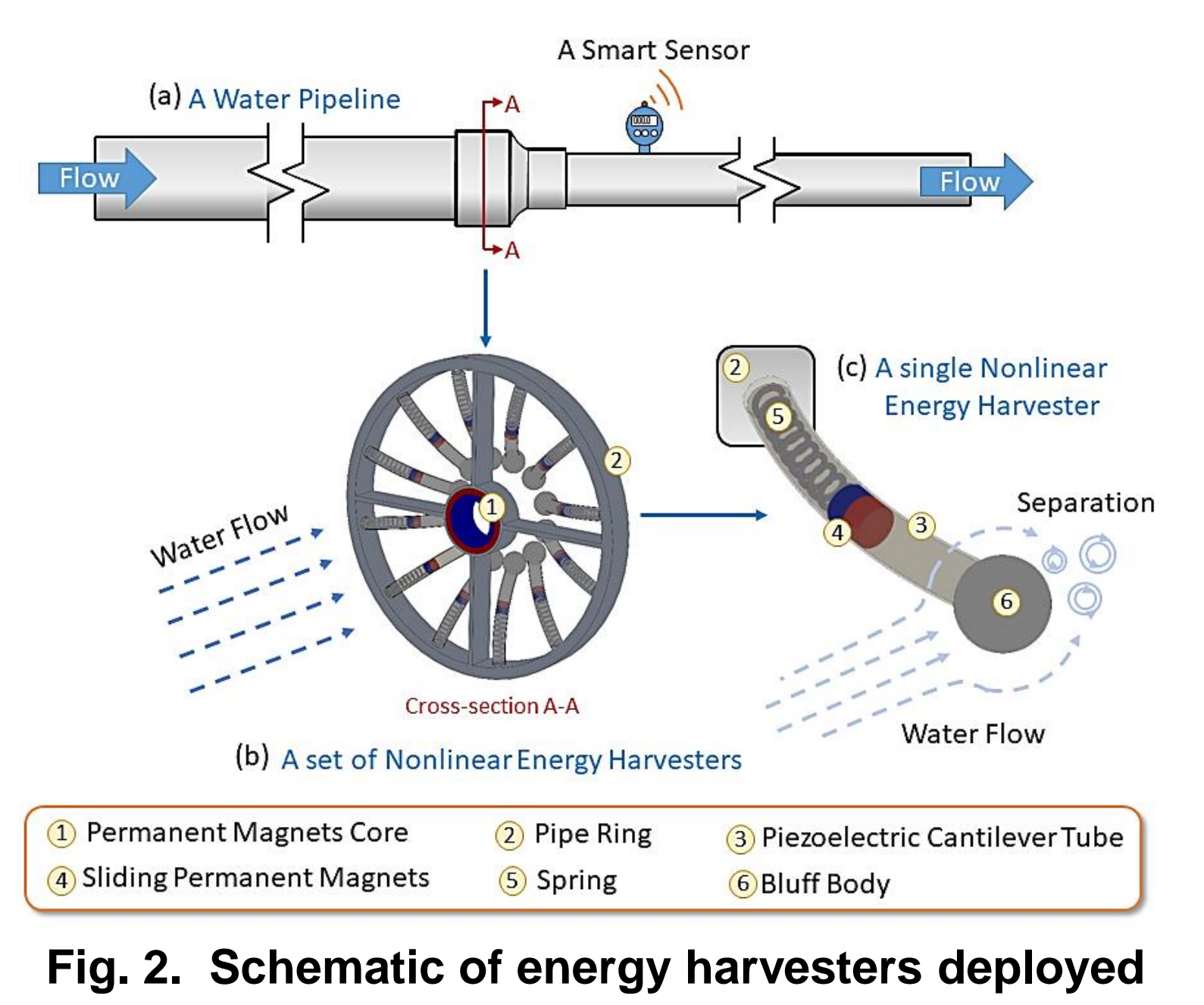

inside a pipe.

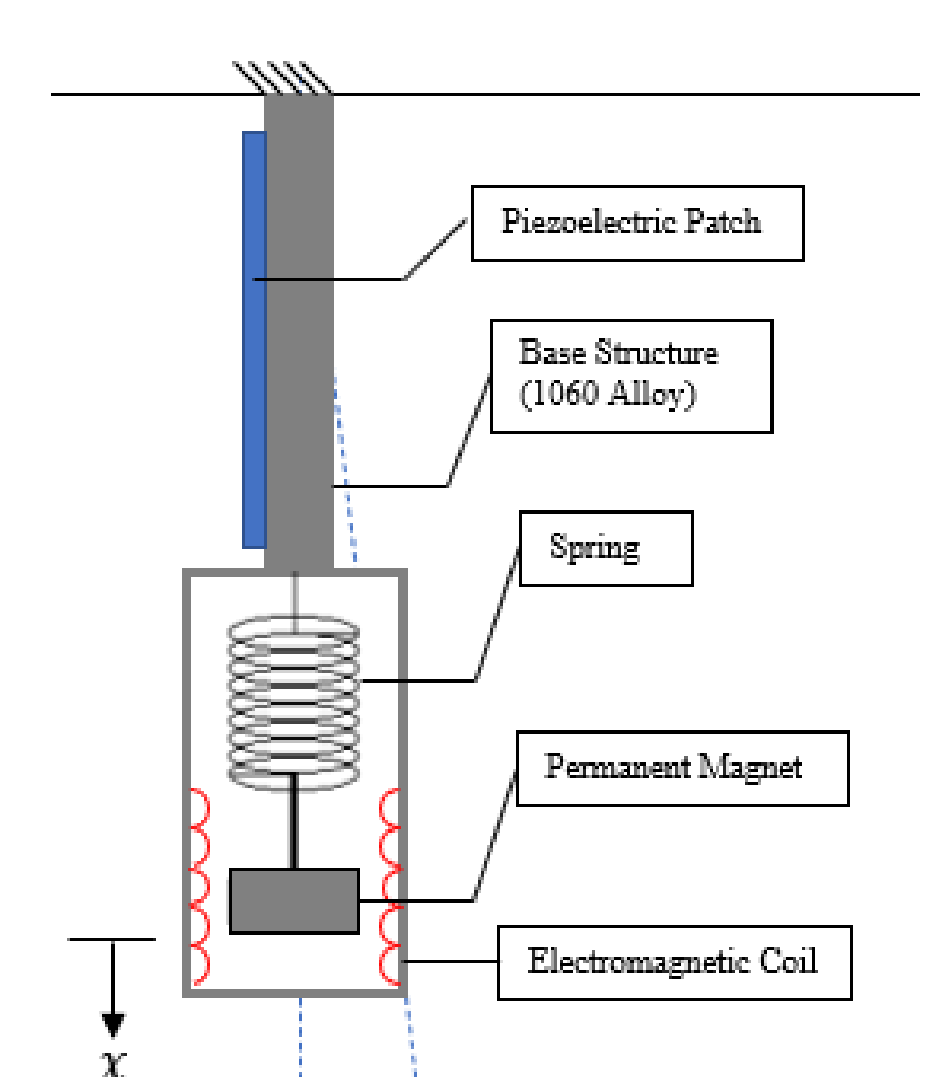

$\theta$

Fig. 3. Proposed Design of Nonlinear Vibration Energy Harvester.

\section{ACKNOWLEDGEMENTS}

This publication was supported by Qatar University Internal Grant no. IRCC2020-017. The findings achieved herein are solely the responsibility of the authors.

\section{FUTURE WORK}

The hybrid energy harvester can is extended to an array that can maximize harvesting efficiency and performance output.

Design and construct an integrated circuit with a wireless sensing node and application to compare the feasibility in real-world applications

\section{EXPERIMENTAL SETUP}

The experimental was conducted to measure the voltage output from the energy harvesters in different configurations. It was fitted on an open channel water tank with a variable speed and water level. Smart Materials Macro-Fiber Composite (MFC) P2-2814 piezoelectric was used for piezoelectric energy harvesting. Electromagnetic energy harvesting used two metal balls inside $0.375 \mathrm{~mm}$ copper wire coils with a total 200 coils. Bluff-body housing and oscillator spring was 3D printed using polyethylene terephthalate glycol (PETG) allowing for a compact shape and ease of manufacturability. Data acquisition was taken on DeweSoft hardware and software with real-time and FFT analysis.

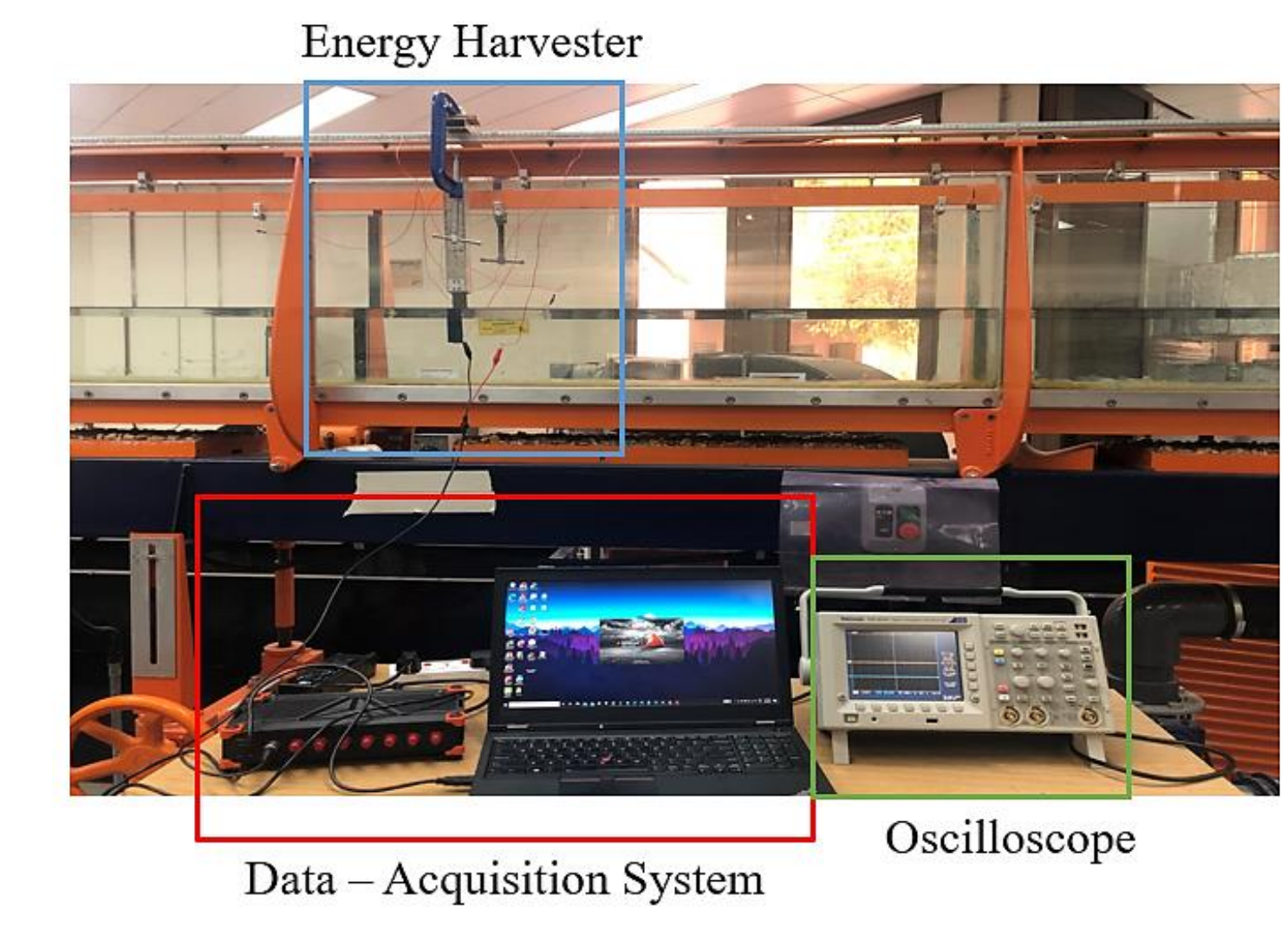

Fig. 4. Open Water Channel Experimental Setup

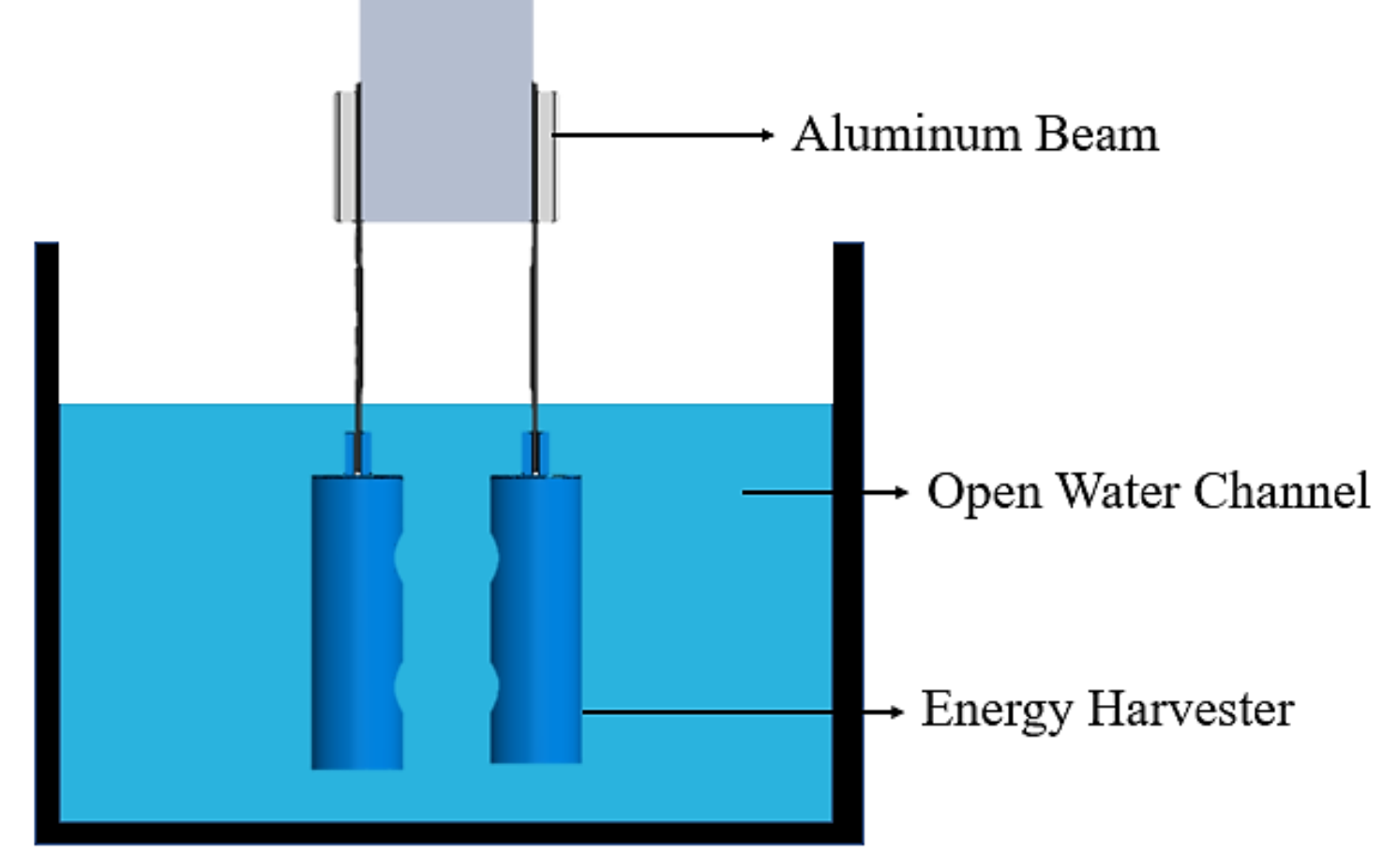
Fig. 5. Assembly of Setup for Dual Energy
Harvesters for Broadband Applications

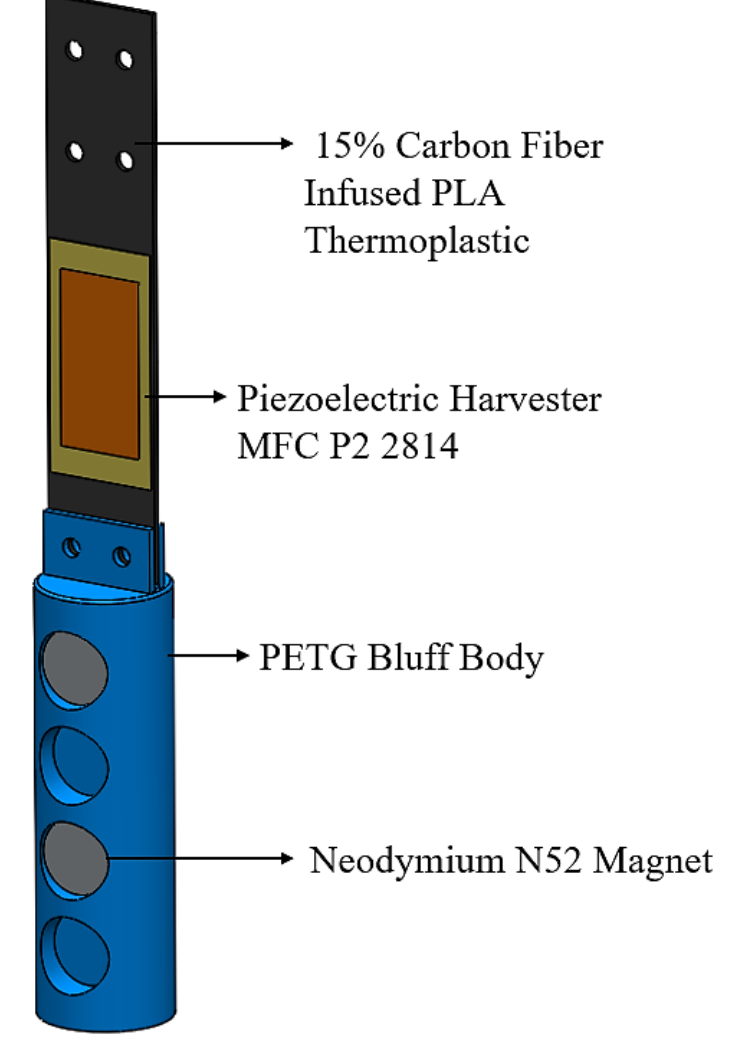

Fig. 6. Schematic of Broadband Piezoelectric Energy Harvester

\section{RESULTS AND DISCUSSION}

Part A - Modelling: An optimization study across different bluff body shapes and performance highlighted that VIV performs better for lower velocities and 'galloping' mechanism outperforms VIV at higher velocities.

Vorticity varied with the bluff body shapes. Ellipse and isosceles triangle-shaped bluff body performed best during resonance in a frequency study with finite element modelling methods [3].

Part B - Optimization: Dual-Mass Configuration models enhanced the overall voltage output compared to a single mass VIV-based harvester in L-configurations between $7 \%$ to $52 \%$.

Tuning the secondary beam for inline oscillations (twice of transverse) was able improve piezoelectric performance by $21 \%$ to $52 \%$ under resonance; L-Vertical with Vertical Beam was the best performer.

Heavier bluff body allows for energy harvesting at lower flow velocities since the synchronization frequency decreases. Relationship of synchronization in water and air was also proposed. Part C - Broadband: The addition of nonlinear magnetic forces can enhance the energy harvesting properties at lower velocities by $600 \%$ and broaden the synchronization range by up to $35 \%$.

- However, narrowband performance near resonance outperforms broadband by $25 \%$ to $55 \%$.

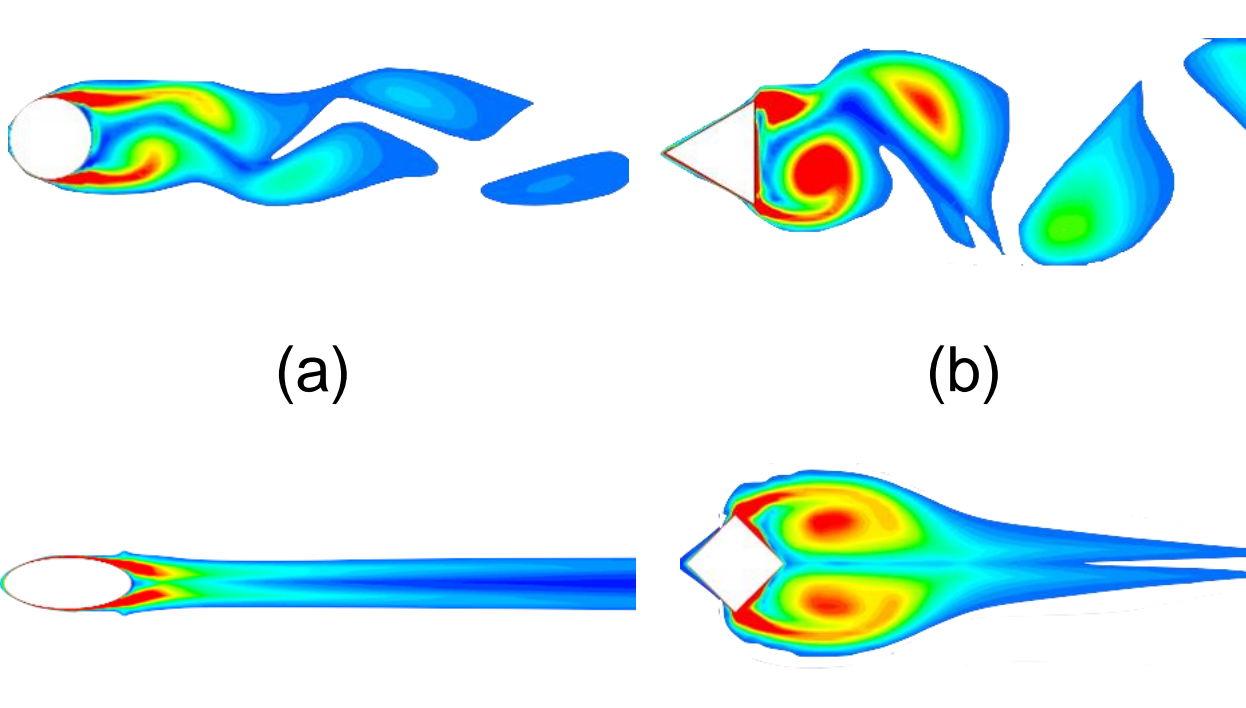

(c) Fig. 7. Vorticity Formation for various bluff
bodies; (a) Cylinder; (b) Triangle; (c) Ellipse; (d) Quadrilateral

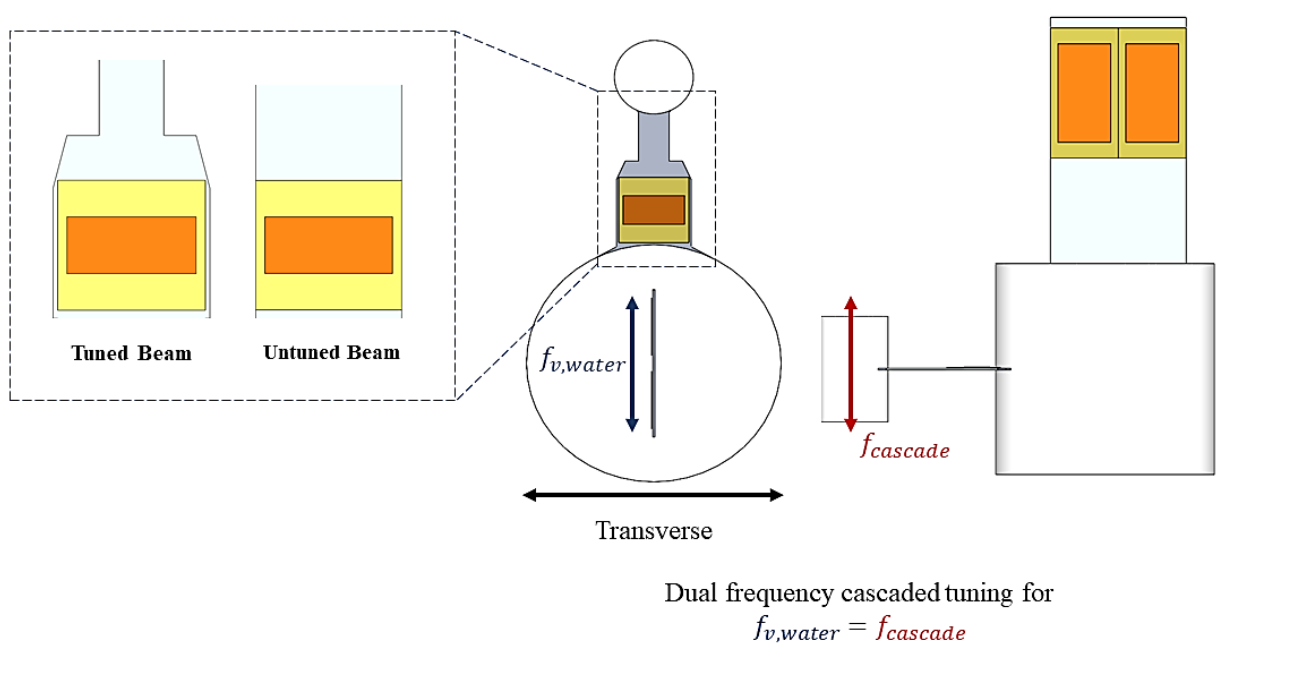
Fig. 8. Vorticity Formation for Various Bluf Bodies; (a) Cylinder; (b) Triangle; (c) Ellipse; Niw Fig. 9. Voltage Output for Dual-Mass Hybrid Energy Harvesters Comparison

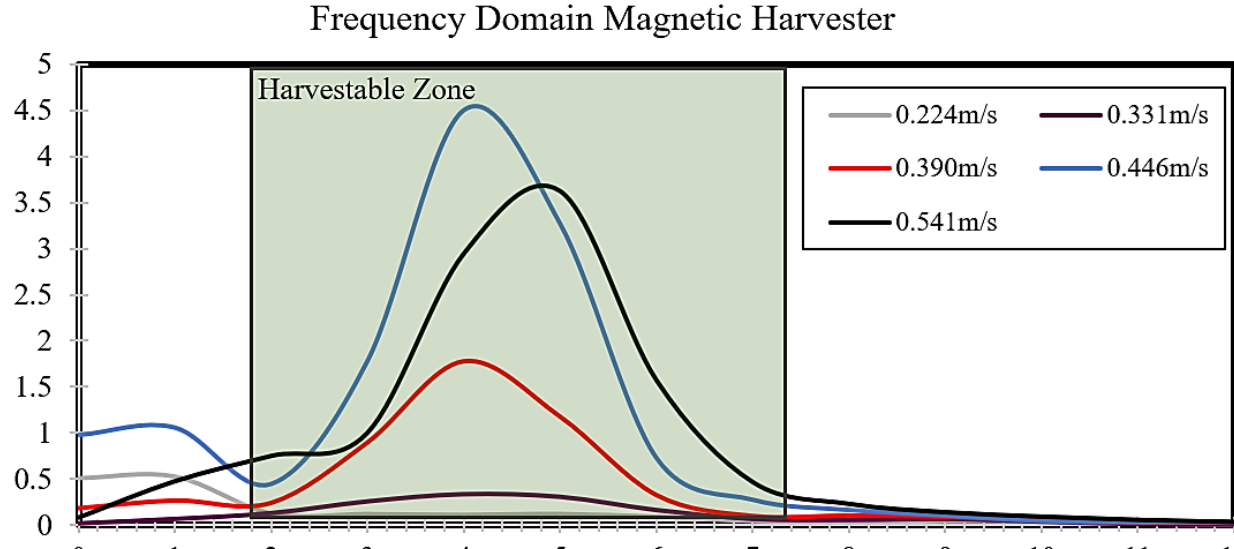

Fig. 10. Frequency Respesponse of Magnetic Broadband Dual Harvester Systen

\title{
5. CONCLUSION
}

The addition of a tip mass provides a housing for an internal electromagnetic oscillator and generates turbulence behind the body which is used for vibration-based energy harvesting.

Hybrid energy harvesters can increase the voltage output compared to conventional methods vibration-energy harvesting, especially for powering up MEMS in remote areas.

A hybrid energy harvester with both piezoelectric and electromagnetic methods can enhance energy harvesting efficiency with the inclusion of a dual-mass design.

Narrowband piezoelectric energy harvesting is more superior for applications of fixed flow and designed to oscillate near the structural natural frequency.

\section{REFERENCES}

1] N.E. Dutoit, B.L. Wardle, S.-G. Kim, Design considerations for mems-scale piezoelectric mechanical vibration energy harvesters, Integr. Ferroelectr. 71 (2005) 121-160. https://doi.org/10.1080/10584580590964574.

2] C.H.K. Williamson, R. Govardhan, A brief review of recent results in vortex-induced vibrations, J. Wind Eng. Ind. Aerodyn. 96 (2008) 713-735. https://doi.org/10.1016/j.jweia.2007.06.019.

[3] M. Hafizh, A.G.A. Muthalif, J. Renno, M.R. Paurobally, M.A. Arab, I. Bahadur, H. Ouakad, A hybrid piezoelectricelectromagnetic nonlinear vibration energy harvester excited by fluid flow, Comptes Rendus Mécanique. 349 (2021) 65-81. https://doi.org/10.5802/crmeca.74.

[4] A.G.A. Muthalif, N.H.D. Nordin, Optimal piezoelectric beam shape for single and broadband vibration energy harvesting: Modeling, simulation and experimental results, Mech. Syst. Signal Process. 54 (2015) 417-426. https://doi.org/10.1016/j.ymssp.2014.07.014 